\title{
Surface effects on friction-induced fluid heating in nanochannel flows
}

\author{
Zhigang Li \\ Department of Mechanical Engineering, The Hong Kong University of Science and Technology, Clear Water Bay, Kowloon, Hong Kong
}

(Received 17 November 2008; published 25 February 2009)

\begin{abstract}
We investigate the mechanism of friction-induced fluid heating under the influence of surfaces. The temperature distributions of liquid argon and helium in nanoscale Poiseuille flows are studied through molecular dynamics simulations. It is found that the fluid heating is mainly caused by the viscous friction in the fluid when the external force is small and there is no slip at the fluid-solid interface. When the external force is larger than the fluid-surface binding force, the friction at the fluid-solid interface dominates over the internal friction of the fluid and is the major contribution to fluid heating. An asymmetric temperature gradient in the fluid is developed in the case of nonidentical walls and the general temperature gradient may change sign as the dominant heating factor changes from internal to interfacial friction with increasing external force. The effect of temperature on the fluid heating is also discussed.
\end{abstract}

DOI: 10.1103/PhysRevE.79.026312

PACS number(s): 47.61.Fg, 47.85.Np, 44.15.+a, 68.35.Af

Fluid motion in nanoconfinements has attracted great interest due to its potential applications in many areas of science and engineering. In nanofluidics, the surface area to volume ratio is high and the surface plays an important role because it affects the physical and dynamic properties of the fluid in complicated ways [1-6]. The velocity profile in nanoscale Poiseuille flows qualitatively follows parabolic distribution, as predicted by the Navier-Stokes (NS) equations. However, the magnitude of the velocity can be considerably different from the estimation of NS equations $[7,8]$ given the pressure drop. In many cases, the flow rate is orders of magnitude smaller than predicted by the continuum theory. This is caused by the strong fluid-surface friction, which also converts external work to internal heat and therefore heats up the fluid. Furthermore, the internal viscous friction due to fluid-fluid molecular collisions can be viewed as another heat source. This heat source is nonuniform because the density of the fluid is inhomogeneous under the influence of the surface. The heat generated by the fluid-fluid and fluid-surface frictions is eventually dissipated through the channel wall and the heat transfer depends on the thermal resistance at the fluid-wall interface, which is related to many factors, such as fluid-surface interaction, the structure of the surface, and boundary conditions [9-12]. These three factors, internal viscous friction, fluid-surface friction, and interfacial thermal resistance, are usually coupled and make the surface effect on the fluid heating a very complex issue.

The sources of friction could cause nonuniform temperature distribution in the fluid, depending on the surface effect. This is true especially for the cases in which the surfaces involve different materials. A temperature gradient in fluids is associated with the phenomena of thermophoresis or thermodiffusion $[13,14]$, in which suspensions tend to concentrate on either the high- or low-temperature side depending on the sign of their thermodiffusivity $[15,16]$. This could promote the possible applications of friction-induced fluid heating for particle and biomolecule separation through micro- or nanochannels $[17,18]$. Attempts have been made to understand the heat transfer in nanochannel fluid flow by using Monte Carlo and molecular dynamics (MD) simulations $[9,11,19,20]$, but little work has been done to understand the heating of confined fluids induced by the sources of friction. Although it is well known that the viscous effect can cause the temperature of confined fluids to increase, the effect of surfaces is not fully understood and the mechanism of friction-induced fluid heating in nanochannel flow is still unclear.

In this work, we use nonequilibrium molecular dynamics (NEMD) simulation to investigate the temperature change in nanoscale planar Poiseuille flows. Temperature variations in liquid $\mathrm{Ar}$ and $\mathrm{He}$ between identical and nonidentical parallel walls are examined. Our attention is focused on the qualitative mechanisms of the surface effect on the fluid heating. It is found that the fluid heating is governed by internal viscous friction of the fluid, at small pressure drop. For large pressure difference, the fluid-surface friction dominates over the internal friction. The thermal resistance at the fluid-wall interface is another critical factor, which restrains the heat dissipation through the wall.

The Poiseuille flow system considered in this work consists of two parallel planar solid walls, which could be of different materials. A liquid, Ar or He, is confined between the walls and an external force is applied to each fluid molecule in the direction parallel to the walls. The material of the wall is $\mathrm{Ag}$ and/or Al. The thermal vibration of the atoms in the wall is considered by using the tight-binding potential function, which has been tested and widely used for transition metals $[21,22]$. The fluid is described by the LennardJones $(\mathrm{LJ})$ potential, $U(r)=4 \varepsilon_{\alpha \beta}\left[\left(\sigma_{\alpha \beta} / r\right)^{12}-\left(\sigma_{\alpha \beta} / r\right)^{6}\right]$, where $r$ is the separation between two interacting atoms of species $\alpha$ and $\beta$, respectively, $\sigma_{\alpha \beta}$ is the collision diameter, and $\varepsilon_{\alpha \beta}$ is the binding energy. The interaction between the fluid molecules and atoms in the wall is also calculated by the LJ potential and the interaction parameters follow the

TABLE I. Lennard-Jones parameters.

\begin{tabular}{lcrc}
\hline \hline Atom (molecule) & $\sigma(\AA)$ & $\varepsilon(\mathrm{K})$ & Reference \\
\hline $\mathrm{He}-\mathrm{He}$ & 2.550 & 10 & {$[23]$} \\
$\mathrm{Ar}-\mathrm{Ar}$ & 3.470 & 114 & {$[23]$} \\
$\mathrm{Ag}-\mathrm{Ag}$ & 2.574 & 4075 & {$[24]$} \\
$\mathrm{Al}-\mathrm{Al}$ & 2.551 & 4736 & {$[24]$} \\
\hline \hline
\end{tabular}




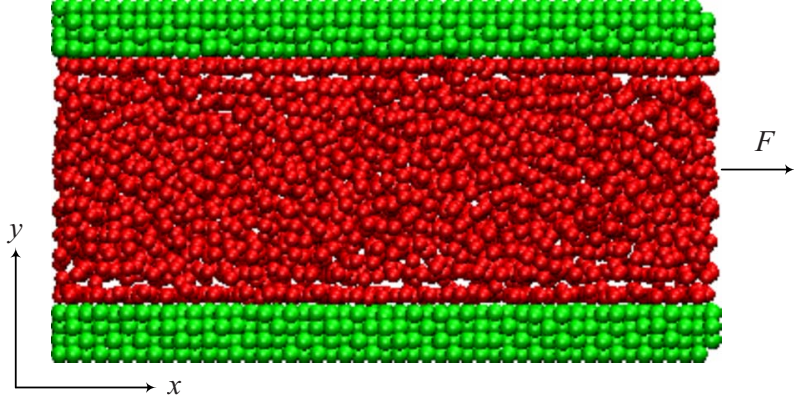

FIG. 1. (Color online) Structure of a typical planar Poiseuille flow system. Outer (green) particles are wall atoms and inner (red) particles are fluid molecules. External force is applied in the $x$ direction.

Lorentz-Berthelot mixing rule, in which the collision diameter $\sigma_{\alpha \beta}$ is given by the arithmetic mean of the selfinteraction parameters of the two species, $\sigma_{\alpha \beta}=\left(\sigma_{\alpha \alpha}\right.$ $\left.+\sigma_{\beta \beta}\right) / 2$, and the binding energy is approximated by the geometric mean, $\varepsilon_{\alpha \beta}=\sqrt{\varepsilon_{\alpha \alpha} \varepsilon_{\beta \beta}}$. In order to implement this mixing rule for interactions, we use the LJ parameters given in Refs. [23,24] for the fluid and wall. The values of $\sigma$ and $\varepsilon$ are summarized in Table I.

The structure of a typical NEMD system is shown in Fig. 1. The walls are parallel to the $x$ axis and each wall consists of four layers of atoms. The distance between the two innermost layers, in the $y$ direction, is $3.7 \mathrm{~nm}$, and the lengths of the system in the $x$ and $z$ directions are 9.8 and $4.9 \mathrm{~nm}$. Periodic boundary conditions are employed in the $x$ and $z$ directions only. The potential is truncated at $10.21 \AA$ and Newton's equations are integrated with Beeman's leapfrog algorithm $[1,22,25]$, with time step equal to 1 fs. Initially, the wall atoms and fluid molecules are on fcc lattice sites and then the system is relaxed for 100 ps to reach equilibrium. After the equilibration, a force is applied to each fluid molecule in the positive $x$ direction. This external force is linearly increased with time until it reaches the desired value in 50 ps. Afterward, the system is allowed to equilibrate for another $100 \mathrm{ps}$ before the temperature distribution and the other properties of the fluid are measured. Due to the interaction between the fluid and surface, the external force on the fluid is eventually transmitted to the walls. Therefore, to prevent the walls from moving, we fix the atoms in the outermost layer of the walls and allow the atoms in the other three inner layers free to vibrate, which are sufficient to account for the effect of surface flexibility on the fluid motion. The Berendsen thermostat is used to maintain the temperature of the wall [26]. In this sense, the wall behaves like a heat sink, which dissipates the energy transferred to the system in the form of work done by the external force. The fluid is divided into 20 bins in the $y$ direction and the positions and velocities of fluid molecules are recorded every ten steps such that a large amount of molecules are available for calculating the temperature. The temperature in the $i$ th bin, $T_{i}$, is calculated based on the peculiar velocity of each molecule relative to the streaming velocity of the molecules in the bin,

$$
T_{i}=\sum_{j=1}^{N_{i}} m\left(v_{i}-\bar{v}\right)^{2} /\left(3 N_{i}-\delta\right) k,
$$

where $m$ and $v_{i}$ are the mass and laboratory velocity of each molecule in the bin, $N_{i}$ and $\bar{v}$ are the total number and mean velocity of the molecules in the bin, $k$ is the Boltzmann constant, and $\delta$ is the number of degrees of freedom used up in determining the mean velocity, which is small compared with $N_{i}$.

We first investigate the temperature variation of liquid $\mathrm{Ar}$ and He between two Ag walls. The temperature of the wall is maintained at $T=100 \mathrm{~K}$. Three different external forces $F_{1}$, $F_{2}$, and $F_{3}$, which cover a wide range, are used to observe the temperature distribution at different situations. The forces and the corresponding pressure drops along the channel are given in Table II. The temperature variation in the fluids is shown in Fig. 2. It is seen that the temperature profile, for all the external forces, generally follows the same fashion that it is uniform around the center area and drops toward the walls. This is expected since all the friction-generated heat has to be dissipated through the two identical walls. Although the temperature distribution looks similar, the mechanism for the fluid heating is different. When the external force is smaller than the fluid-surface binding force, the stick boundary condition applies and there is no relative motion between the fluid and wall at the interfaces, as illustrated by the velocity profile in Fig. 3(a). Hence, the temperature increase in Fig. 2(a) is mainly caused by the viscous friction in the fluid. It is noted that the viscous friction in the fluid is nonuniform because the density of the fluid is inhomogeneous due to surface effects, as shown in Fig. 3(b). This nonuniform viscous friction can be reflected in the temperature profile if the walls are nonidentical, as discussed later.

As the external force is increased [Fig. 2(b)], the friction in the fluid becomes relatively strong and heats up the fluid further. In Fig. 2(b), it is also seen that the temperature gap between the fluids and wall is larger than the case of small external force. To explain the gap, it is necessary to measure the thermal resistance $R_{\mathrm{th}}$ between the fluid and surface. There are several ways to obtain the thermal resistance [27]. In this work, $R_{\mathrm{th}}$ is determined as the ratio of the temperature gap $\Delta T$ at the interface to the heat flow $q$ through the wall. In the simulations, the total kinetic energy $E$ of the vibrating atoms in the wall and the difference between this total energy

TABLE II. External force and the corresponding pressure drop along the channel.

\begin{tabular}{lccc}
\hline \hline & \multicolumn{3}{c}{ Force in MD simulation [pressure drop (MPa)] } \\
Fluid & $F_{1}$ & $F_{2}$ & $F_{3}$ \\
\hline Argon & $2.5 \times 10^{-4}(70.9)$ & $1.0 \times 10^{-3}(284)$ & $3.0 \times 10^{-3}(851)$ \\
Helium & $5.0 \times 10^{-5}(12.7)$ & $2.0 \times 10^{-4}(50.8)$ & $6.0 \times 10^{-4}(152)$ \\
\hline \hline
\end{tabular}



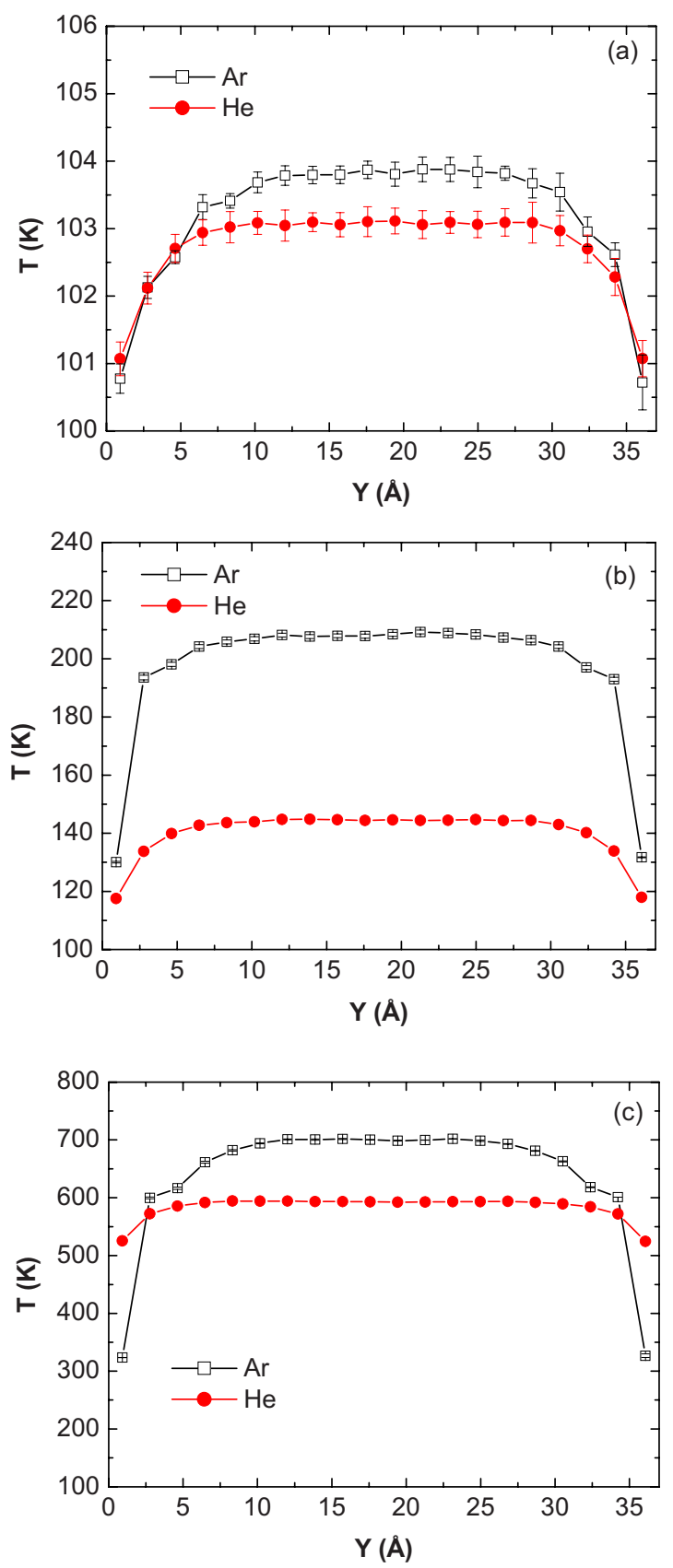

FIG. 2. (Color online) Temperature distributions in liquid Ar and He between two $\mathrm{Ag}$ walls of $100 \mathrm{~K}$ under different external force, (a) $F_{1}$, (b) $F_{2}$, and (c) $F_{3}$. Error bars in (b) and (c) are well below $0.5 \%$.

and the desired wall temperature, $\Delta E=E-3 N k T / 2$, are calculated at each step before the thermostat is applied, where $N$ is the number of vibrating atoms. The heat flow through the wall is estimated as the average of the energy difference, $q=\langle\Delta E\rangle$. The interfacial thermal resistance in the cases of Figs. 2(a) and 2(b) are equal to $R_{\mathrm{th}}=1.18 \pm 0.06 \times 10^{-9}$ and $R_{\mathrm{th}}=1.10 \pm 0.04 \times 10^{-9} \mathrm{~m}^{2} \cdot \mathrm{K} / \mathrm{W}$ for $\mathrm{Ar}$ and $R_{\text {th }}$ $=1.60 \pm 0.01 \times 10^{-9}$ and $R_{\mathrm{th}}=2.30 \pm 0.04 \times 10^{-9} \mathrm{~m}^{2} \cdot \mathrm{K} / \mathrm{W}$ for He, which are of the same order. However, the heat generation $q$ in the case of Fig. 2(b) is more than that of Fig. 2(a). Based on Fourier's law of conduction, $q=\Delta T / R_{\mathrm{th}}$, the temperature gap for the case of Fig. 2(b) has to be large to conduct away enough heat.

If the external force is increased further, the temperature of the fluid increases greatly but the shape remains almost the same. In addition, a big jump is found in the temperature at the interface, as shown in Fig. 2(c). In this case, the external force becomes larger than the fluid-wall binding force and there is a slip at the fluid-wall interface, as indicated by the velocity profiles in Fig. 3(a). The friction at the interface due to the slip is the major contribution to the heat generation because the fluid-wall interaction is much stronger than the fluid-fluid interaction. For relatively small external forces, the interfacial thermal resistance for $\mathrm{Ar}$ is smaller than that for He because Ar-Ag interaction is stronger than He-Ag interaction. With the slips, it is obtained that $R_{\text {th }}$ for Ar does not change too much $\left(R_{\mathrm{th}}=0.71 \pm 0.01\right.$ $\left.\times 10^{-9} \mathrm{~m}^{2} \cdot \mathrm{K} / \mathrm{W}\right)$; however, $R_{\mathrm{th}}$ increases considerably at the He-Ag interface $\left(R_{\mathrm{th}}=6.69 \pm 0.01 \times 10^{-9} \mathrm{~m}^{2} \cdot \mathrm{K} / \mathrm{W}\right)$. Therefore, the large temperature gap at the interfaces is caused by the heat generation for Ar, while both heat generation and $R_{\mathrm{th}}$ are important to the temperature jump for He. But for both fluids, the heat induced by fluid-wall friction dominates over fluid-fluid friction. This can also be confirmed by the flows between nonidentical walls, as will be discussed later. In Fig. 2 , it is also found that the surface effect reaches further in the fluid for Ar than He. This is because the Ar-Ag binding is stronger than the He-Ag interaction. It is noted that the temperature profiles of fluids between two identical walls have been investigated through MD simulations [28,29]. It was shown that the temperature distribution carries both quadratic and quartic characters if the spatial variations of the fluid properties are considered [28]. For the temperature profiles in Fig. 2, it is found that the pure quartic term can fit the data very well and the quadratic contribution is not evident. This might be the consequence of small channel size and different molecular interactions in the wall [29].

In Fig. 2, the two walls are made of same material and the temperature profile is symmetric. If the materials of the walls are different, the interactions between the fluid and two walls may compete with each other, redistribute the fluid molecules, and bring about different temperature profiles. Figure 4 shows the temperature variation under the same external forces but the upper wall is changed to $\mathrm{Al}$, which has stronger binding force with the fluids than $\mathrm{Ag}$, based on the values of the binding energy in Table I. The difference in the surface wettability causes asymmetric fluid density distribution. Close to the upper wall (Al), the fluid density is higher and the viscous effect is stronger relative to the fluid around the lower wall. This is consistent with the temperature distribution at small external force shown in Fig. 4(a), in which an asymmetric temperature gradient is developed and it is generally positive in the $y$ direction. It is also seen, in Fig. 4(a), that the temperature for $\mathrm{He}$ is higher than the case of identical walls [Fig. 2(a)]. This is because the thermal resistance $R_{\text {th }}$ at the $\mathrm{He}-\mathrm{Al}$ interface is larger than that at the $\mathrm{He}-\mathrm{Ag}$ interface in Fig. 2(a). Furthermore, in this case, $R_{\text {th }}$ at the lower interface (He-Ag) is also larger than the case of identical Ag walls because the fluid density close to the Ag wall decreases due to the strong $\mathrm{He}-\mathrm{Al}$ interaction at the upper wall. Actually, the same situation happens with Ar. However, 

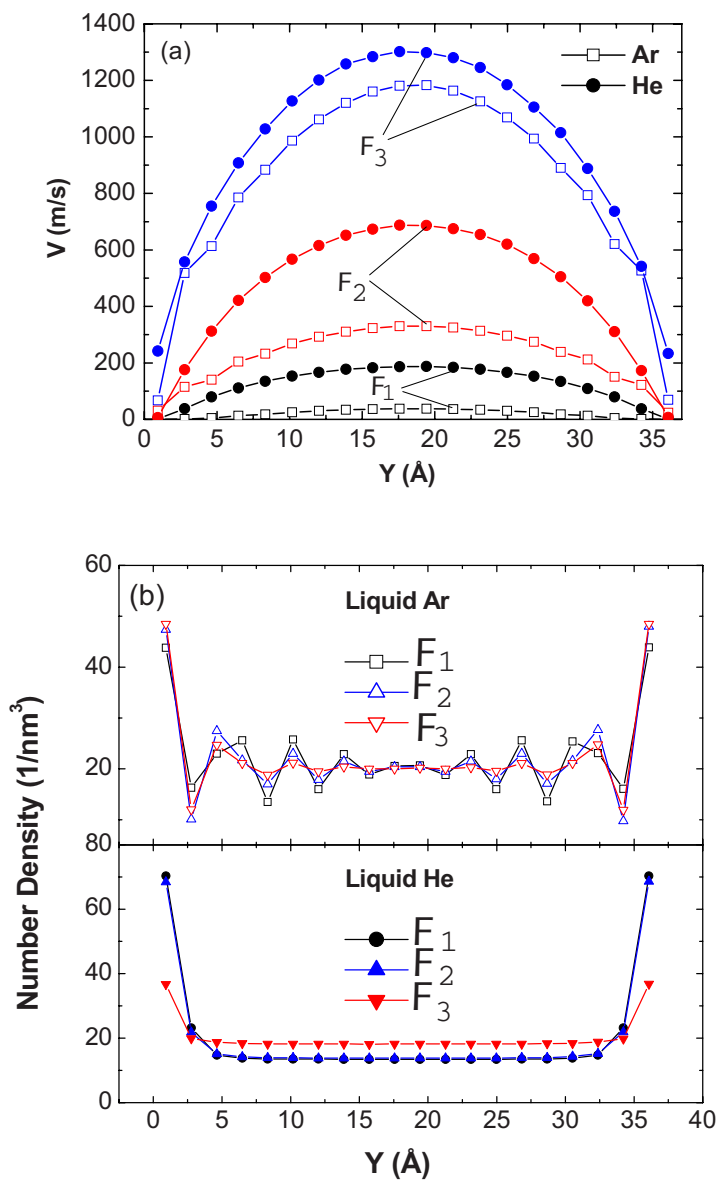

FIG. 3. (Color online) (a) Velocity and (b) density distributions for liquid $\mathrm{Ar}$ and $\mathrm{He}$ between two $\mathrm{Ag}$ walls of $100 \mathrm{~K}$ under external forces $F_{1}, F_{2}$, and $F_{3}$.

the intermolecular interaction among Ar molecules is much stronger than for $\mathrm{He}$; therefore, $\mathrm{He}$ is more sensitive than $\mathrm{Ar}$ to the changes in the surface.

Further increase in the external force enhances the friction in the fluids, and slip may occur depending on the fluidsurface interaction and the structure of the surface $[11,12]$. As the force is increased, the velocity slip takes place first at the lower surface $(\mathrm{Ag})$ for $\mathrm{He}$ and the fluid-surface friction grows to be a dominant factor for heat generation, as illustrated by the negative temperature gradient for $\mathrm{He}$ in Fig. 4(c). For Ar, a slight slip is also observed in Figs. 4(b) and 4(c), but the friction in the fluid seems to be still significant since the temperature in the region close to the upper wall (Al) is higher than that close the lower Ag surface. The velocity distributions corresponding to Fig. 4 are shown in Fig. 5 , where the fluid slips are illustrated. The sign change of the temperature gradient in $\mathrm{He}$ confirms that the friction at the fluid-wall interface dominates the heat generation if a slip takes place.

We have also studied the temperature fluctuation in $\mathrm{Ar}$ and $\mathrm{He}$ in the same channels at wall temperature $T=300 \mathrm{~K}$. The temperature effect lies in the competition between the kinetic energy of the fluid molecules and the fluid-wall binding energy. If the temperature of the system, $k T$, is smaller than fluid-wall binding energy $\varepsilon$, adsorption of fluid mol-
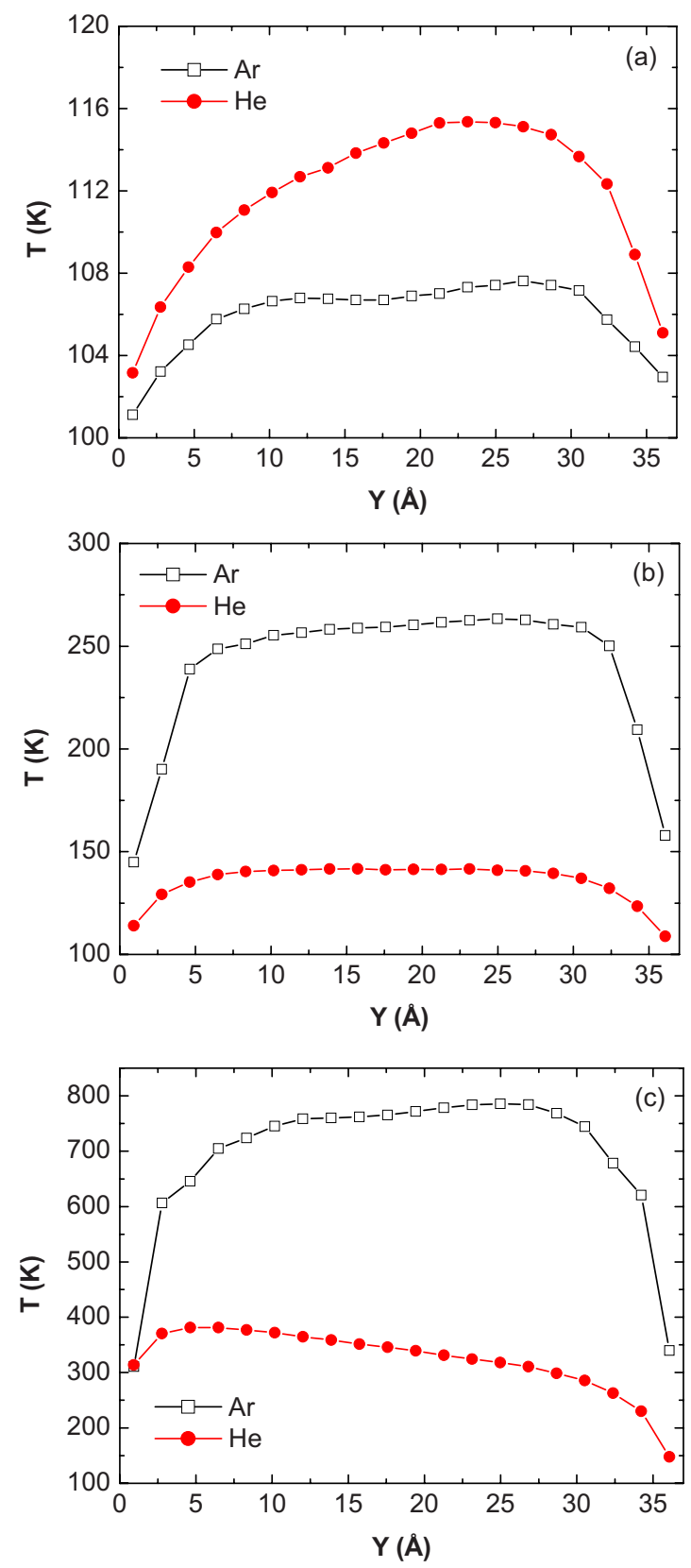

FIG. 4. (Color online) Temperature distributions in liquid Ar and He between two nonidentical walls of $100 \mathrm{~K}$ under different external forces (a) $F_{1}$, (b) $F_{2}$, and (c) $F_{3}$. The lower and upper walls are $\mathrm{Ag}$ and $\mathrm{Al}$, respectively.

ecules on the solid surface is favored; otherwise, the fluid molecules have relatively large kinetic energy to overcome the attraction of the walls and adsorption may not occur [22]. Figure 6 illustrates the temperature variation in the fluids for the cases shown in Figs. 2 and 4 except that the wall temperature is $300 \mathrm{~K}$. Generally, the temperature profiles of the fluids are quite similar to those shown in Figs. 2 and 4. The only difference is that the slip tends to occur at intermediate external force for $\mathrm{He}$. This is because the $\mathrm{He}-\mathrm{Ag}$ and $\mathrm{He}-\mathrm{Al}$ binding energy $\varepsilon \sim 200 \mathrm{~K}$, which is smaller than the surface temperature of $300 \mathrm{~K}$, as is seen in Fig. 6(b2), where the external force is medium but the slip at the He-Ag surface causes the temperature of $\mathrm{He}$ to increase in the area close to 


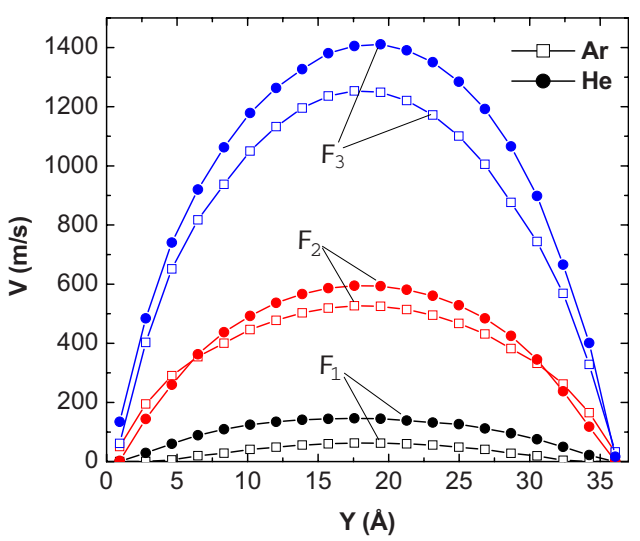

FIG. 5. (Color online) Velocity distributions for liquid Ar and He between two nonidentical walls under external forces $F_{1}, F_{2}$, and $F_{3}$, corresponding to Fig. 4.

the lower surface. Fluid slip at solid surfaces depends on the ratio of $k T / \varepsilon$. Although the effect of temperature on the slip is not significant, the enhanced thermal vibration of wall atoms at relatively high temperature can reduce the ordering of fluid layers near the surface and increases the slip length $[30,31]$. For liquid $\mathrm{Ar}$, the temperature profile remains almost the same because the Ar-Ag and Ar-Al binding energy $\varepsilon$ $\sim 700 \mathrm{~K}$ is much larger than $300 \mathrm{~K}$. Therefore, the increase in the temperature from 100 to $300 \mathrm{~K}$ does not change the fundamental Ar-surface interaction.

In summary, we have investigated the fluid heating in nanoscale Poiseuille flow between two identical and nonidentical planar surfaces. It has been found that the temperature increase in the fluid is determined by the frictions in the fluid and at the fluid-wall interface. For small external force, at which the stick boundary applies, the heating of fluid is mainly caused by the internal friction of the fluid. However, the friction at the fluid-surface interface is the major contribution to heat generation at large external force, which brings about velocity slip at the boundary. Furthermore, the thermal resistance at the interface determines the temperature gap between the fluid and surface as well as the temperature in the fluid. In the channel of identical surfaces, the temperature profile is symmetric. However, the temperature gradient across the channel width becomes asymmetric when the materials of the walls are different. This is because the internal friction is nonuniform and the frictions due to slip at the interfaces are different. The effect of temperature has also been studied and it has been found that the temperature of
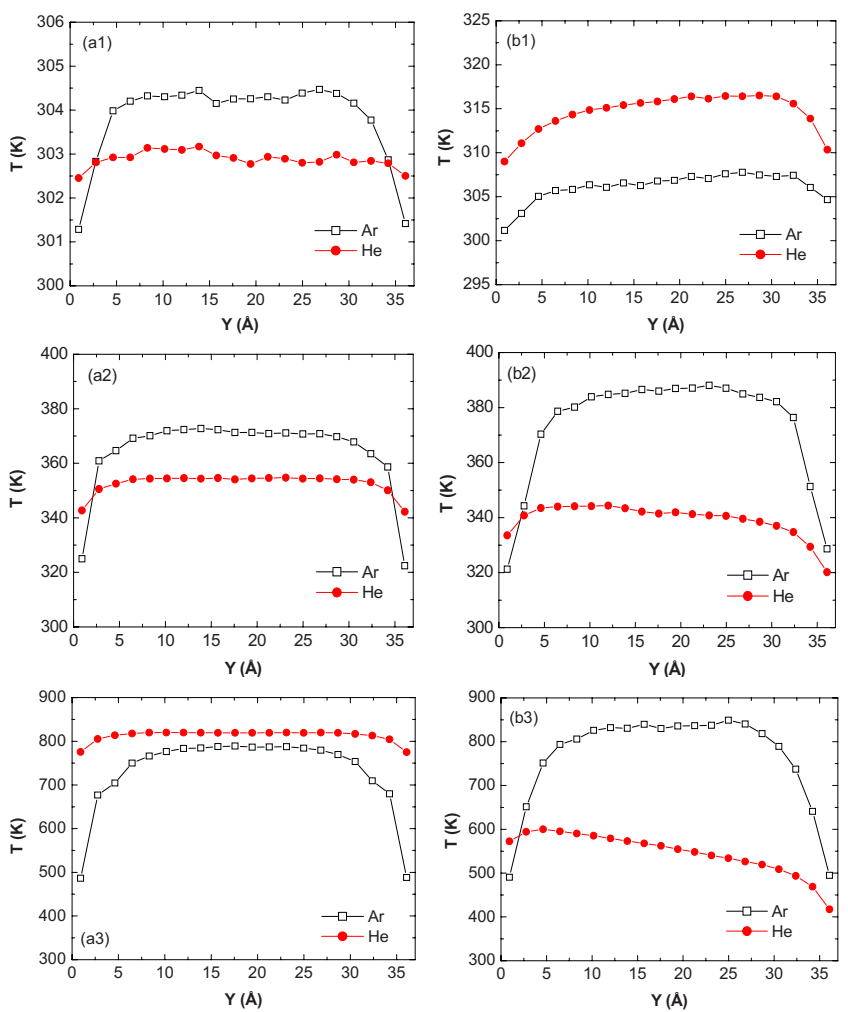

FIG. 6. (Color online) Temperature distributions in liquid Ar and He between two identical (a) and nonidentical (b) walls of $300 \mathrm{~K}$ under different external forces (a1), (b1) $F_{1}$, (a2), (b2) $F_{2}$, and (a3), (b3) $F_{3}$. In (a), the two walls are made of Ag. In (b), the lower and upper walls are $\mathrm{Ag}$ and $\mathrm{Al}$, respectively.

the surface affects the slip at the interface. If the fluid-wall binding energy is smaller than the surface temperature, slip tends to occur at relatively small external force and the friction at the interface becomes important. However, the temperature of the surface has little effect on the temperature profile of the fluid when the fluid-wall binding energy is larger than the surface temperature. Finally, it is noted that the numerical analyses in this work are by no means complete. Some issues, such as how the channel size and geometric and mechanical properties of the surface affect the temperature distribution of the fluid, are still unclear, and the potential application of fluid heating for particle separation is also worthy of investigation.

This work was supported by the Hong Kong Innovation and Technology Fund under Grant No. GHP/035/07GD.
[1] Z. Li and L. Hong, J. Chem. Phys. 127, 074706 (2007).

[2] Z. Li and G. Drazer, Phys. Fluids 18, 117102 (2006).

[3] I. Bitsanis, J. J. Magda, M. Tirrell, and H. T. Davis, J. Chem. Phys. 87, 1733 (1987).

[4] J. Koplik, J. R. Banavar, and J. F. Willemsen, Phys. Fluids A 1, 781 (1989).

[5] R. F. Cracknell, D. Nicholson, and N. Quirke, Phys. Rev. Lett. 74, 2463 (1995).
[6] E. Akhmatskaya, B. D. Todd, P. J. Daivis, D. J. Evans, K. E. Gubbins, and L. A. Pozhar, J. Chem. Phys. 106, 4684 (1997).

[7] H. Takaba, Y. Onumata, and S. Nakao, J. Chem. Phys. 127, 054703 (2007).

[8] C. Huang, P. Y. K. Choi, K. Nandakumar, and L. W. Kostiuk, J. Chem. Phys. 126, 224702 (2007).

[9] A. J. Markvoort, P. A. J. Hillers, and S. V. Nedea, Phys. Rev. E 71, 066702 (2005). 
[10] S. Maruyama and T. Kimura, Therm. Sci. Eng. 7, 63 (1999).

[11] M. Shibahara, M. Katsuki, T. Kunugi, and K. Muko, Nanoscale Microscale Thermophys. Eng. 10, 197 (2006).

[12] R. Khare, P. Keblinski, and A. Yethiraj, Int. J. Heat Mass Transfer 49, 3401 (2006).

[13] R. B. Bird, W. E. Stewart, and E. N. Lightfoot, Transport Phenomena (Wiley, New York, 2002).

[14] Z. Li and H. Wang, Phys. Rev. E 70, 021205 (2004).

[15] E. Blums, S. Odenbach, A. Mezulis, and M. Maiorov, Phys. Fluids 10, 2155 (1998).

[16] B. J. de Gans, R. Kita, and B. Muller, J. Chem. Phys. 118, 8073 (2003).

[17] T. M. Squires and S. R. Quake, Rev. Mod. Phys. 77, 977 (2005).

[18] R. Khare, M. D. Graham, and J. J. de Pablo, Phys. Rev. Lett. 96, 224505 (2006).

[19] M. Wang and Z. Li, Phys. Rev. E 68, 046704 (2003).

[20] G. Nagayama and P. Cheng, Int. J. Heat Mass Transfer 47, 501 (2004).
[21] F. Cleri and V. Rosato, Phys. Rev. B 48, 22 (1993).

[22] Z. Li and H. Wang, Phys. Rev. Lett. 95, 014502 (2005).

[23] H. Hippler, J. Troe, and H. J. Wendelken, J. Chem. Phys. 78, 6709 (1983).

[24] P. M. Agrawal, B. M. Rice, and D. L. Thompson, Surf. Sci. 515, 21 (2002).

[25] D. Beeman, J. Comput. Phys. 20, 130 (1976).

[26] M. P. Allen and D. J. Tildesley, Computer Simulation of Liquids (Oxford University Press, New York, 1987).

[27] P. K. Schelling, S. R. Phillpot, and P. Keblinski, Phys. Rev. B 65, 144306 (2002).

[28] B. D. Todd and D. J. Evans, Phys. Rev. E 55, 2800 (1997).

[29] K. P. Travis and K. E. Gubbins, J. Chem. Phys. 112, 1984 (2000).

[30] P. A. Thompson and M. O. Robbins, Phys. Rev. A 41, 6830 (1990).

[31] R. S. Voronov, D. V. Papavassiliou, and L. L. Lee, Ind. Eng. Chem. Res. 47, 2455 (2008). 